\title{
WWW database of optical constants for astronomy
}

\author{
Th. Henning ${ }^{1}$, V.B. Il'in ${ }^{2}$, N.A. Krivova ${ }^{2}$, B. Michel $^{1}$, and N.V. Voshchinnikov ${ }^{2}$ \\ 1 Astrophysical Institute and University Observatory, Friedrich Schillier University, Schillergäßchen 3, D-07745 Jena, Germany \\ 2 Astronomical Institute, St. Petersburg University, Bibliotechnaja pl. 2, St. Petersburg 198904, Russia
}

Received November 2, 1998; accepted January 24, 1999

\begin{abstract}
The database we announce contains references to the papers, data files and links to the Internet resources related to measurements and calculations of the optical constants of the materials of astronomical interest: different silicates, ices, oxides, sulfides, carbides, carbonaceous species from amorphous carbon to graphite and diamonds, etc.

We describe the general structure and content of the database which has now free access via Internet: http://www.astro.spbu.ru/JPDOC/entry.html or http:// www. astro.uni-jena.de/Users/database/entry.html
\end{abstract}

Key words: astronomical data base: miscellaneous scattering — radiative transfer — molecular data

\section{Introduction}

The optical constants of materials - refractive indices or dielectric permittivities - are widely used in astrophysics. They are input parameters required for any simulations of the light scattering by dust grains and the calculations of radiative transfer in dusty media (galaxies, interstellar clouds, circumstellar shells, protoplanetary disks, atmospheres of brown dwarfs). Since the 60's the volume of the data has been permanently growing and presently it is difficult to overlook the results obtained within about 40 years.

We attempted to collect and classify the data available and to create a place in the Internet for further accumulation of such data.

\section{The database}

The main topics of the database are the optical constants of materials involved in astronomical calculations. These materials are:

Send offprint requests to: V.B. Il'in, e-mail address: vi2087@vi2087.spb.edu
- different kinds of amorphous/glassy/crystalline terrestrial/solar-system/artificial silicates;

- silicon, $\mathrm{SiO}$, crystalline/fused quartz;

- metals: Fe, Mg, etc.;

- oxides: $\mathrm{FeO}, \mathrm{Fe}_{2} \mathrm{O}_{3}, \mathrm{Fe}_{3} \mathrm{O}_{4}, \mathrm{MgO}, \mathrm{Al}_{2} \mathrm{O}_{3}, \mathrm{MgAl}_{2} \mathrm{O}_{4}$;

- sulfides: FeS, MgS, $\mathrm{SiS}_{2}$;

- carbides: SiC, TiC;

- carbonaceous species: amorphous carbon, glassy carbon, HAC, coals, graphite, diamonds;

- organics: tholin, kerogen, "organic refractory";

- $\mathrm{H}_{2} \mathrm{O}, \mathrm{CO}, \mathrm{CO}_{2}, \mathrm{NH}_{3}, \mathrm{HCN}$ and other ices.

The database includes references to the papers, data files and links to the Internet resources related to measurements, calculations, compilations, or approximations of the optical constants.

\subsection{References}

The database contains about 500 references to the papers, books, dissertations where the refractive indices, reflectance, transmittance, mass absorption coefficient, and so on were derived. Most of the papers were published in the time interval from the 60's till 1998.

\subsection{Data}

We have started filling the database with the data available for us. They are the optical constants obtained in the laboratory of Astrophysical Institute of Friedrich Schiller University (Jena) as well as the data derived in the Soviet Union and some often used freely accessible data.

It should be noted that the Jena data were intended especially for astronomical applications. They cover a wide spectral range, show the dependence on important parameters: $\mathrm{Fe} / \mathrm{Mg}$ ratio, temperature, etc. Besides, well prepared samples and sophisticated control were used in these measurements (see, e.g., Jäger et al. 1994; Jäger et al. 1998; Schnaiter et al. 1998).

Several papers on the optical constants of the materials under consideration were published in former Soviet 
Union journals (see Zolotarev et al. 1984 for a review). As these journals are not easily accessible, we scanned the data tables/figures in the papers printed before 1974 (when the Soviet Union joined the international copyright agreement) and put them in our database.

The data we included are presented in their initial format (table or figure) and in our standard tabular format. In some cases we also give plots of the refractive index vs. wavelength and dielectric permittivity vs. energy dependences and an information on how the data were obtained, what equipment was used and what samples were considered.

The service codes of the database allow to transfer the data from our standard format to a desirable form (refractive index/dielectric permittivity vs. wavelength/wavenumber/energy table with numbers of any format). Our light scattering codes based on Mie theory and Separation of Variables Method for spheroids give the possibility to transform the data into cross-sections, emissivity and so on.

\subsection{Related topics}

References to some useful books and reviews on the subject (e.g. the classical handbooks by Palik 1998, 1991) and links to Internet collections of optical data files and personal WWW pages with related software were also placed in the database.

First of all, among these Internet resources one should mention the WWW database of the optical constants in the X-ray domain created at the Lawrence Berkeley National Laboratory (http://www-cxro.lbl.gov/optical_ constants), and the collections of the data files obtained for a very large number of ice mixtures at the Leiden University (http://www.strw.leidenuniv.nl/ lab) and for amorphous carbon by V. Zubko (http://physics. technion.ac.il/ zubko/nk.html). There are also useful tools for the Kramers-Kronig analysis and Effective Medium Theory available in the World Wide Web.

Many light scattering codes have been developed and there are two general lists of such codes in the Internet. Besides links to these lists, we also give direct links to personal WWW pages where one can find most popular codes to simulate the electromagnetic radiation scattering by spheres (Mie theory), spheroids (Separation of Variables Method), axisymmetric particles (T-matrix Method), irregular-shaped particles (Discrete Dipole Approximation) and others.

\subsection{Some details}

Anyone can freely access the database - its Internet address is http://www.astro.spbu.ru/JPDOC/entry.html (server in St. Petersburg) or http://www.astro.uni-jena. de/Users/database/entry.html (server in Jena).

First, one will arrive at a welcome WWW page with a short description of the database and a link to the database homepage. The latter includes our list of the material groups (silicates, oxides and so on) and a description of related topics (Internet resources, books, etc.).

Each group of materials has its own page. It contains a table whose columns show: the state of material (amorphous, crystalline, glassy), the quantity measured or calculated (refractive index, reflectance, etc.), the form of the data (table, figure), the considered wavelength range (in microns), and reference to the paper.

When the data in a paper were available we created a corresponding WWW page and supplied the table with a link to the page. Such pages can contain links to data and graphic files, information about the paper (e.g. its ADS abstract), a description of how the data were derived and so on.

\section{Concluding remarks}

We plan to continue filling in the database and developing its service. Certainly, many good papers and related Internet resources were missed by us. Therefore, we shall be very thankful to anyone who could send us more references, data files, or links to be included in the database. Any comments and remarks are also welcome. Our contact address isvi2087@vi2087.spb.edu.

Acknowledgements. The authors acknowledge the financial support of the Volkswagen Foundation (Germany) that made this work possible.

\section{References}

Jäger C., Mutschke H., Begemann B., Dorschner J., Henning Th., 1994, A\&A 292, 641

Jäger C., Mutschke H., Henning Th., 1998, A\&A 332, 291

Palik E.D. (ed.), 1985, Handbook of optical constants of solids. Academic Press, Boston

Palik E.D. (ed.), 1991, Handbook of optical constants of solids II. Academic Press, Boston

Schnaiter M., Mutschke H., Dorschner J., Henning Th., Salama F., 1998, ApJ 498, 486

Zolotarev V.M., Morozov V.N., Smirnova E.V., 1984, Optical constants of natural and technical media. Leningrad, Khimija (in Russian) 